\title{
Developing Verification and Optimization Models for Corona Discharge Suppression in High Voltage AC and DC Capacitor Banks
}

\author{
M. J. Javadi
}

\begin{abstract}
Capacitor banks are widely used in current electrical transmission systems in order to improve power quality and increase efficiency. Utilizing high voltage components such as, shunt capacitors in the power grid imposes new challenges to the system which are required to be addressed. One of these challenges is corona discharges that can have negative impacts on capacitor banks such as power loss, insulator erosion followed by equipment failure, and radio interference. Although previous studies have almost exclusively focused on optimization of corona suppression rings for transformers and transmission lines, no specific studies have conducted regarding high voltage capacitor banks. This paper presents a novel study concerning verification and development of corona discharge suppression models on AC and DC capacitor banks with two different voltage levels. The employed method is based on the Maxwell's equations and finite element method (FEM) which is implemented with the help of COMSOL Multiphysics $\odot$ software. Results have verified the necessity of suppression methods as well as the efficiency of proposed solutions. Corona inception voltage levels are identified and effective factors on its appearance are reviewed. Analyses of proposed solutions have shown significant improvements in optimization of corona suppression methods as well as enhancement of maintenance maneuverability.
\end{abstract}

Index Terms-Corona discharge, corona ring, corona suppression, high voltage capacitor banks, shunt capacitor banks

\section{INTRODUCTION}

$\mathrm{C}$ APACITOR banks are widely used in AC and DC power systems in order to compensate reactive power. Due to the inductive effect of loads and transmission equipment (transformers and transmission lines), additional reactive power $(\mathrm{Q})$ is required to flow in the grid due to the lagging power factor. This reactive power cannot be produced from the generation point since the capacity of the power system will decrease, voltage will drop and power loss will increase. In order to address this problem, IEEE standard 1036 introduced shunt capacitors as an optimal solution for reactive power compensation. These capacitors reduce power losses and increase grid capacity by preventing reactive power flow through the entire system as it is produced locally. In other words, shunt capacitors are being installed near loads in transmission and distribution substations. As it is illustrated by the blue arrow in Fig. 1, reactive power $(\mathrm{Q})$ surges to the load from an AC capacitor bank in the way that it is compensated locally rather than from the generation point [1]. Consequently, power losses are reduced substantially in the grid as well as fuel consumption for power generation in fossil fuel-based power plants.

DC capacitor banks are used extensively in high voltage direct current (HVDC) systems. Long distance power transmissions as well as renewable power plants are connected via HVDC systems to the power grid. These banks are normally installed in converter stations at the end of each HVDC link in order to maintain voltage stability. Apart from the capacitor bank type (AC or DC), integration of these components in high voltage power systems may introduce new challenges such as corona discharges.

Corona is a common and undesirable phenomenon in high voltage power transmission systems which mainly depends on electric field strength and distribution [2]. Insulator gradual degradation due to the surface bombardment with discharged ions, equipment failures, power losses, and radio interferences are some of the disadvantages of corona discharges [3]. As a part of the solution, increasing the apparent radius of active area and reducing the electric potential gradient is essential for prevention of its inception. A common solution that has been used in industry is utilization of corona suppression rings that cover the critical areas by reducing the electric field gradient on the active surface and structural elements where the electric field is extremely high.

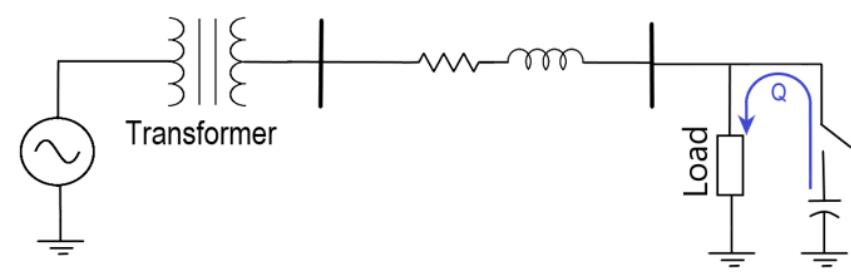

Fig. 1. Reactive power compensation from AC capacitor bank to the load point, based on the solution proposed by IEEE standard 1036.

M. J. Javadi, is with Environment and Energy Department, Mälardalen University, Box 883, $72123 \quad$ Västerås, Sweden (email:mj223wb@student.lnu.se). 
Various studies have conducted regarding the corona suppression rings on insulators which are used on transmission lines and transformers [4]-[7]. Mohan [8], investigated the corona ring optimization on various high voltage transmission lines and concluded that tube thickness, distance from high voltage end, and ring radius are three important factors that can be modified in order to enhance the performance of the ring. Terrab and Kara [9], proposed alterations on the tube thickness and ring dimensions regarding $230 \mathrm{kV}$ insulators. Furthermore, dimensional changes on the ring is proposed as a part of optimization by Barros et al. [10]. However, no specific studies have conducted regarding the corona effect on capacitor banks. Therefore, this paper is a novel study regarding the corona discharge behavior on AC and DC high voltage capacitor banks based on the verification models and optimized solutions. Proposed models are compared and analyzed with the current designs. Significant improvements were achieved in terms of corona suppression and maintenance maneuverability. This paper consists of 5 sections. Section 2 describes briefly the required theoretical background based on the previous studies as well as different types of corona discharges (negative and positive). Effective factors on inception of corona discharges are reviewed from various studies and differences between AC and DC corona inception voltages are reflected. Section 3 consists of the employed methods and simulation of $500 \mathrm{kV}$ AC and $500 \mathrm{kV}$ DC capacitor banks with the help of COMSOL Multiphysics@ software. The simulation process and considered factors are explained in detail as well as corona inception voltage threshold. Section 4 presents the simulation results and discussion. The critical areas of the current corona suppression methods are identified and proper solutions are proposed. A comparison is carried out between the current and proposed models in order to demonstrate the optimization and efficiency of the ring structural alteration. Finally, the last section consists of conclusions.

\section{THEORETICAL BACKGROUND}

Although in the absence of electric field, gas molecules behave as an insulator, by exposing them to a sufficiently strong electric field their behavior changes to conductive due to the formation of low impedance path as a result of free charges generation and ionizations [11].

\section{A. Physics of Corona Discharge}

The process of ionization depends on free electrons in the surrounding gas. A free electron can be created as a result of three phenomena, photon impact, electron impact, and detachment. On the other hand, free electrons also can be lost during the attachment process with other atoms. Thus, either negative ions or neutral molecules can be created due to the recombination with positive ions. The number of free electrons under the electric field can be calculated based on the subtraction of lost electrons from the generated ones by (1) [2].

$n_{e}(t)=n_{0} \exp \left[\left(v_{i}-v_{a}\right) t\right]$

where $t$ is time, $v_{i}$ is ionization frequency, $v_{a}$ is attachment frequency, and $n_{0}$ is the gas constant. This equation shows that once $v_{i}$ exceeds $v_{a}$, number of electrons increase exponentially with the time which is known as avalanche. It can be calculated that the required electric field strength for avalanche initiation in the air at atmospheric pressure (AP) is $3 \mathrm{kV}$ [12]. Creation of an individual avalanche cloud chamber is the initial behavior of the air which is followed by secondary avalanches. The secondary avalanches are created based on the emitted photons as a result of collisions between positive ions (which are moving towards the cathode) and electrons (which are moving towards the anode). Accumulation of such avalanches increase the primary chamber strength to the level that streamers are generated [2], [13], [14]. When an electron changes the energy level, it absorbs or releases photon in the form of light. Therefore, the emitted visible light during the corona discharge occurrences is due to the electron recombination with ions [2], [15]. Radiated photons are absorbed by more atoms which results in creation of more electrons known as secondary avalanches [16].

\section{B. Type of Corona Discharges}

Identifying various types of corona discharges is essential in order to explain the behavior of electric field at each rack of capacitor banks further in section 4. Corona discharge is categorized in two groups, positive (anode) and negative (cathode). Kuffel et al. [3] and Chang et al. [17], broadly investigated various types of corona discharges which details of their experimental studies and electric field calculations is beyond the scope of this paper and only results are reflected here. As shown in Fig. 2, by increasing the DC voltage on positive rod, streamers initiate to appear. By rising the voltage, self-sustained streamers are created as a result of equality between the number of positive ions generated by primary avalanche, and secondary avalanche. This transition towards steady glow is called positive corona which occurs before the complete breakdown [16]. Negative corona occurs when the rod is loaded with negative charges. In this situation, a fastpropagating glow or concentrated tiny points (known as tufts or beads) emerge instead of streamers [18]. In general, negative corona propagation relies on the gas molecule ionizations while, positive corona requires photoionization in order to propagate. Furthermore, breakdown and corona inception voltages are lower for positive corona due to the considerable number of ions produced near the anode in comparison with cathode [19], [20]. Table 1, summarizes the differences

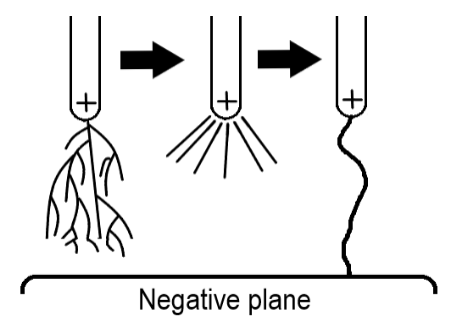

(a) Positive corona discharge

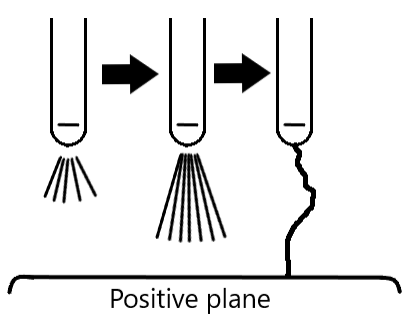

(b) Negative corona discharge
Fig. 2. Positive and negative corona discharge stages [3]. 
between positive and negative corona discharges.

TABLE I

DIFFERENCES BETWEEN POSITIVE AND NEGATIVE CORONA DISCHARGES

\begin{tabular}{c|c|c}
\hline \hline Characteristic & Positive Corona & Negative Corona \\
\hline Corona inception voltage & Low & High \\
\hline Appearance & Glow & Tiny points \\
\hline Reason of occurrence & Photoionization & Molecule ionization \\
\hline
\end{tabular}

\section{Effective Factors on Corona Discharge Appearance}

Apart from the type of discharge (corona or breakdown), several factors have considerable impacts on corona inception namely, polarity, humidity, surrounding gas pressure, geometry, voltage, and pollution. An experimental study regarding the wind effects on DC positive corona conducted by D.Ariza et al. [21]. The study was implemented for eight voltage levels between 5 and $12 \mathrm{kV}$ with three various air velocities. Results have shown that wind speed does not have any effect on corona onset voltage although, the corona current increases with the wind velocity. Results of another investigation by $\mathrm{C}$. Hsu and $\mathrm{C}$. Wu [11] have shown that by increasing the air flow rate from 0 to $50 \mathrm{~m} / \mathrm{s}$, the transition voltage rises from $10 \%$ to $20 \%$. However, an erratic behavior was expressed during an experimental study on HVDC systems conducted by J.Kuffel et al. [22] i.e., wind can either ignite or extinguish corona discharges.

1) Polarity

J.Riba et al. [19] studied visualization of corona on AC and

DC voltages and concluded that positive DC corona appears in lower voltages in comparison with AC. An electric field which is generated by DC is steadier and more sustained than AC. Therefore, exposed air atoms and molecules to such field are ionized in lower voltages. Moreover, alternating field created by AC $(50 \mathrm{~Hz})$ causes slower ionization process since electrons are getting repelled and attracted constantly during the wave transition from halfnegative cycle to half-positive [19]. Consequently, under the alternating voltages the complete breakdown occurs consistently in the positive cycle of the wave [3].

2) Pressure

The relationship between pressure and breakdown voltages studied by Leob et al. [23] and Paschen [24]. Paschen's curve demonstrates the relation between the breakdown voltages, gap distances, and pressures for different type of the gases. In higher altitudes where the air pressure is low, discharges (corona and breakdown) occur in lower voltages.

3) Humidity

Humidity is one of the factors that has a significant impact on corona appearance and it is defined as any incident that rises the number of drifting water molecules in the air such as fog and rain. An experimental study was conducted by Zhang et al. [25] for different DC voltage levels in various humidity conditions. Measurements and calculations on this study was based on the number of released photons per second which shows the severity of corona discharges. Results have shown that by maintaining the voltage and rising the humidity levels from $45 \%$ to $85 \%$, number of released photons increases by $40 \%$ which shows the growth of corona discharge intensity. This effect can be explained by the rise of ignited avalanches as a result of emitted photons. Based on calculations, escalation of emitted photons from 500 per-meter to 1000 per-meter results in corona inception voltage reduction from $12.59 \mathrm{kV}$ to 12.48 $\mathrm{kV}$ [16]. Since the drift velocity of ions in humid air is lower than the dry air, less charges flow towards the ground and the possibility of having impact between ions and free electrons (which results in photon generation) rises [26]. However, for voltages below $70 \mathrm{kV}$ the humidity impact is not significant [25].

4) Pollution

Pollution can be generated from various sources such as sea salt transferred by wind, industrial air pollutants, road salts, and bird excrement. Pollutants can be divided into two categories, covering contaminants and floating contaminants. Covered areas with a layer of pollution are neutral as long as the surface is dry. However, a light rain, fog, or mist can turn the pollution layer to a conductive path which increases the electric field non-uniformity. Also, air pollution has a considerable impact on discharges as the suspended particles can be charged and create a conductive paths in the air [3].

\section{MethodS AND Simulation Models}

Due to the complex nature of corona discharge and geometry of high voltage capacitor banks, finite element method (FEM) is applied in this study by utilizing COMSOLC Multiphysics software in order to calculate electric field and identify critical areas with high values on two different capacitor banks. The simulation was implemented based on the Maxwell's equations and finite element method. The complementary method is boundary element method (BEM) which is used in order to define the proper mesh on the model boundaries and solve partial differential equations. In other words, a volumetric finite element mesh can be integrated with BEM-based physics. One of the significant advantages of this method is mesh adjustability and flexibility in critical areas where high simulation accuracy is required and mesh can be defined relatively smaller in comparison with other geometry parts. Simulations are implemented for two different capacitor banks with various voltage levels, $500 \mathrm{kV}$ AC and $500 \mathrm{kV}$ DC. Capacitor banks are simulated once without corona suppression rings to identify the critical electric field areas and once with them in order to evaluate their effects on the model. The same approach is utilized for the proposed solutions in order to assess their impacts on the gradient of the electric field.

\section{A. Boundary Conditions}

Boundary conditions are applied on the geometric entities in order to segregate the interior region of the geometry from unspecified outside areas as well as interfaces within the same geometry. Applying these conditions on the hollow 3D objects 
is limited to the surface edges. The highest voltage values can be calculated on the surface and the E-field strength reduces considerably after few centimeters in the air. Thus, the boundary condition is applied on the surface of the capacitor banks rather than interior boundaries. Boundary condition consists of material type, material property, relative permittivity, potential (applied voltage), pressure, and temperature.

\section{B. Corona Inception Threshold}

The first step in order to evaluate the existence of corona discharges is to calculate the electric field and to detect the areas with highest electric field intensities. These regions should be compared with the limits and in case of exceeding the threshold, corona discharges occur. The necessary condition for a discharge formation is positive effective ionization. However, this condition is insufficient unless an electron avalanche is integrated with it. Assume that the formation of self-sustained avalanche is fulfilled. The next requirement is to maintain effective ionization factor positive along the field lines by calculating effective ionization $\left(\alpha_{e f f}\right)$ for air by using the following equation.

$$
\alpha_{e f f}(E)=p\left[k(E / p-\Lambda)^{2}-A\right]
$$

where $k=1.6\left(\mathrm{~mm} \mathrm{bar} / \mathrm{kV}^{2}\right), \Lambda=2.2(\mathrm{kV} / \mathrm{mm}$ bar $), \mathrm{A}=0.3$ ( $\mathrm{I} / \mathrm{mm}$ bar), and $p$ is pressure (bar). By solving (1), it is concluded that for standard atmospheric pressure (1 bar), electric field values should be equal to $2.6 \mathrm{kV} / \mathrm{mm}$ or higher. In this condition positive effective ionization $\left(\alpha_{\text {eff }}>0\right)$ can be created. Thus, the critical E-field for corona appearance in the air is $2.6 \mathrm{kV} / \mathrm{mm}$ without considering environmental factors such as humidity and pollution [20], [27].

\section{High Voltage Capacitor Banks}

Two capacitor banks with $500 \mathrm{kV}$ AC and $500 \mathrm{kV}$ DC voltage levels were selected to be modeled. The field values which are equal or higher than $2.6 \mathrm{kV} / \mathrm{mm}$ are considered as corona discharge areas. Fig. 3, demonstrates an AC capacitor bank and corona rings at the top three levels based on the corresponding voltages. The HV busbar is installed at the top of the capacitor bank while, the LV busbar is located at the bottom. Each level has some units which are connected in series and the voltage decreases linearly based on the unit capacities. The supporting rack, capacitor unit boxes, and corona rings are in contact with each other as well as the cable which connects the middle terminals of all the units located on each level. Thus, they have the same potential and each rack is isolated from the other with proper insulators as well as the bottom rack from the ground.

Some factors are considered in each simulation regardless of the bank type or geometry as follow:

- The chosen study is electrostatic (es) and stationary.

- The ground is modeled with a large plane under the lowest level insulators.

- Insulators are considered as silicon with relative permittivity of 11.7 and they are simplified with cylinders.

- For more proper meshing, diameter of the connecting cables is considered as $18.88 \mathrm{~mm}$ which is two times larger than the actual size. Therefore, the calculated electric field for cables should be multiplied by the factor of two.

- The default temperature is considered $20^{\circ} \mathrm{C}$ and default pressure is 1 atm for all models.

\section{1) $500 \mathrm{kV} \mathrm{AC} \mathrm{Bank}$}

Fig. 3, demonstrates a $500 \mathrm{kV}$ AC capacitor bank consists of 6 levels where 16 units are located on each level. Each two adjacent units are parallel with each other and all the groups are in series on each side of the bank i.e., each circuit includes 24 groups of tow-by-two parallel units. The potential decreases over each group of units by $6 \mathrm{kV}$. Connecting cables and terminals are shielded with corona rings at top three levels. Voltage is converted from phaseto-phase into phase-to-ground and the incoming voltage is $288 \mathrm{kV}$ which is distributed linearly across the units in the way that the last terminal has $144 \mathrm{kV}$. Distance between the ground and lowest edge of the rack is $2.6 \mathrm{~m}$. The air domain dimension is $33 \mathrm{~m} \times 32 \mathrm{~m} \times 25 \mathrm{~m}$ and it is selected in the way that does not include any free charges. Cables, terminals, HV busbar, and corona rings are meshed with elements less than $7 \mathrm{~mm}$. In order to simplify calculations, frames are filled with a block and unified with units. Since capacitor shells and frames have the same potential, unifying them does not interfere with accuracy.
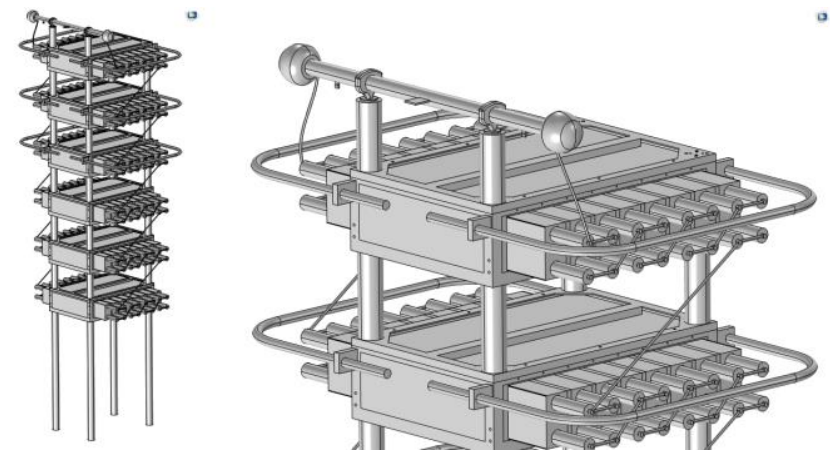

Fig. 3. $500 \mathrm{kV}$ AC capacitor bank with corona rings at top three levels

\section{2) $500 \mathrm{kV} \mathrm{DC} \mathrm{Bank}$}

Fig. 4, illustrates a $500 \mathrm{kV}$ DC capacitor bank with 16 levels and 64 units. The bank is symmetrical due to the even distribution of the units on each level and it consists of two parallel circuits with 32 units in series. The top seven levels are shielded with corona rings and voltage distribution is linearly from the $\mathrm{HV}$ terminal by $500 \mathrm{kV}$ to the $\mathrm{LV}$ terminal by $20 \mathrm{kV}$. The voltage drop across each unit is $15 \mathrm{kV}$. Due to the symmetrical geometry of the bank and in order to reduce the number of created meshes and calculations, the bank is segregated in to two equal parts and calculations are implemented on one part. The distance between the lowest level and ground is $0.7 \mathrm{~m}$ and the air domain dimension is $32 \mathrm{~m} \times 32 \mathrm{~m} \times 29 \mathrm{~m}$. Cables, terminals, and rings are meshed with free tetrahedral elements less than $7 \mathrm{~mm}$ which 
resulted in creation of more than 10.7 million elements on the entire geometry.
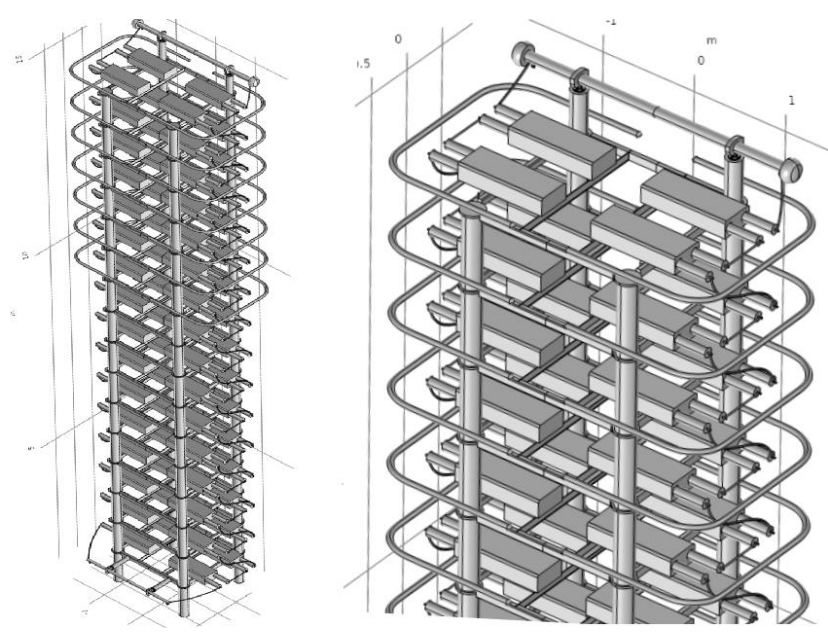

Fig. 4. $500 \mathrm{kV}$ DC capacitor bank with corona rings at top seven levels

\section{Simulation ReSUlts AND Discussion}

E. Kuffel et al. [3] stated that corona inception voltage can be reduced considerably among the bundle of conductors in comparison with a single cylindrical conductor. This implies the interactions between the conductors and it is considered as the foundation of corona suppression rings in capacitor banks. However, the efficiency of these rings should be assessed. Therefore, presented simulation results consist of the following scenarios for each capacitor bank:

- Bank model without corona rings in order to identify the critical areas with high electric field intensities

- Bank model with corona rings in order to evaluate the efficiency and necessity of the rings

- Bank model with proposed solutions in order to compare with previous models and evaluate the efficiency.

\section{A. $500 \mathrm{kV}$ AC Bank}

Fig. 5, demonstrates the surface electric field strength and distribution for $500 \mathrm{kV}$ AC bank without corona rings. By filtering electric field values less than corona inception threshold $(2.6 \mathrm{kV} / \mathrm{mm})$, critical areas can be identified with the red color as shown in Fig. 6. Maximum electric field at the surface of unit shells and frames are $1.8 \mathrm{kV} / \mathrm{mm}$ and 0.7 $\mathrm{kV} / \mathrm{mm}$ respectively. Therefore, these elements are considered as non-critical areas as they are extremely below the limit while, top-level cables show corona discharges, specifically the first connection between the HV busbar and the first terminal. In order to conceive the variation of electric field along the entire capacitor bank levels from ground to the first terminal, it is essential to create a Cut Line $3 D$ and measure the field values as a function of distance from the ground, Fig. 7. Top-level cables are overstressed and E-field values exceed $2.6 \mathrm{kV} / \mathrm{mm}$ (red dashed line). Since there is a direct relationship between the E-field and voltage, the field becomes stronger as distance from the earth increases. However, the field strength in each level decreases substantially few centimeters out of the surface. In other words, the energy of the E-field is absorbed by air molecules.

The next scenario is the simulation of the bank with corona rings in order to verify the effectiveness of these rings on elimination of discharges. Fig. 8, depicts the surface electric field strength and distribution for $500 \mathrm{kV}$ AC bank with corona rings. Results have shown electric field severity reduction and corona discharge elimination. Another effective visualization technique that creates an important insight of the electric field behavior is equipotential line figure at surrounding areas of the geometrical boundaries. Thus, the same conclusion can be achieved from the equipotential lines as shown in Fig. 9. The intensity of the E-field is measured by concentration of the lines and their color range. It is observed that equipotential lines are closer and highly concentrated around the cables in the bank without rings rather than the model with rings. In addition, considerable low E-field values (below $2 \mathrm{kV} / \mathrm{mm}$ ) alongside the bank confirms the effect of the rings as shown in Fig. 10.

\section{B. $500 \mathrm{kV}$ DC Bank}

The simulation results for $500 \mathrm{kV}$ DC bank without suppression rings are presented in Fig. 11. Top-level cables are extremely stressed due to the high potentials applied on them. The E-field intensity decreases linearly from the top due to the voltage drop on each level. By considering the red dashed line as the corona inception threshold, several critical areas are identified. All the cables and terminals located on the top four levels have shown extremely high E-field values. From the $5^{\text {th }}$ until the $9^{\text {th }}$ level, corona discharges emerge on one side. This phenomenon can be explained by positive and negative corona. According to table 1 in section 2, positive corona appears on lower voltages rather than negative corona. As it is shown in Fig. 12, one side of the cable with the potential of $350 \mathrm{kV}$ has $-15 \mathrm{kV}$ in comparison with adjacent terminal and rack which is considered as negative corona and does not show any discharges while, the same cable on the next level turns to positive corona since it has $+15 \mathrm{kV}$ in comparison with adjacent terminals. As a result, positive corona appears on one side.

As shown in Fig. 13, suppression rings have eliminated corona discharges except at the top level with the potential of $485 \mathrm{kV}$. Thus, for capacitor banks with extremely high voltage levels, suppression rings cannot control discharges effectively.

\section{Proposed solutions}

Based on the previous simulations, two solutions were proposed and evaluated. Results have shown significant improvements in elimination of corona discharges as well as enhancement of maintenance maneuverability due to the free spaces created in front of the units. It is concluded that integration of both solutions simultaneously, is an optimal solution for corona discharge suppression in any type of capacitor bank. Proposed solutions are explained as follow:

1. The middle part of the ring does not have substantial effect on the performance of it. Therefore, a new ring is proposed as shown in Fig. 14. Simulation results have shown efficiency of the 


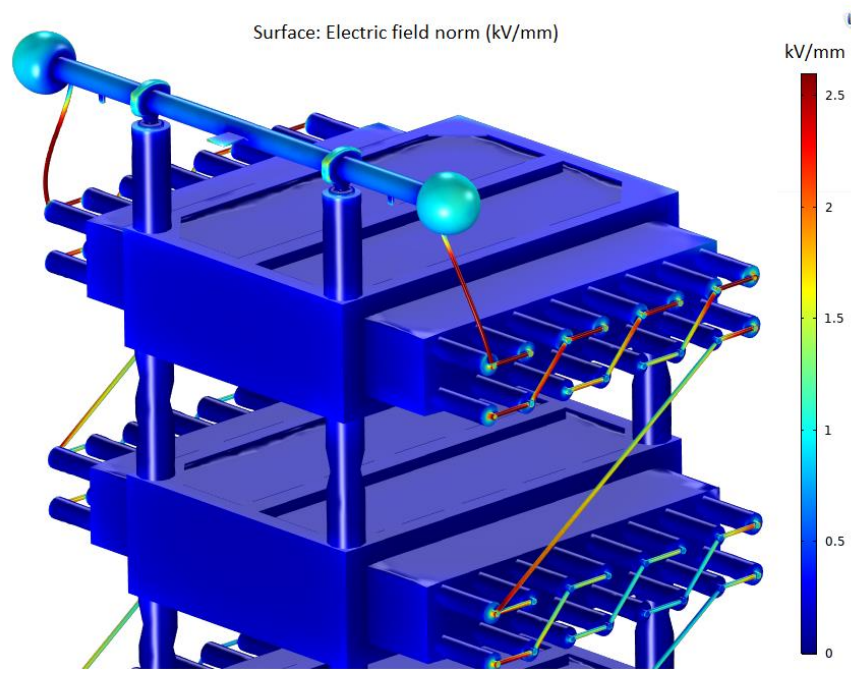

Fig. 5. Electric field distribution for $500 \mathrm{kV}$ AC capacitor bank without corona rings

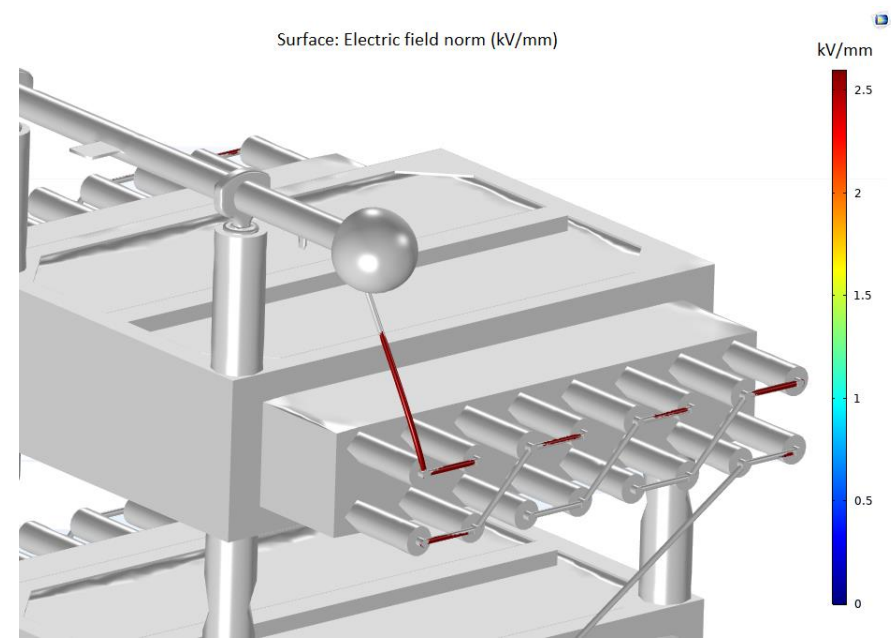

Fig. 6. Intensive electric field areas with values above corona inception threshold $(2.6 \mathrm{kV} / \mathrm{mm})$ for $500 \mathrm{kV}$ AC capacitor bank without corona rings

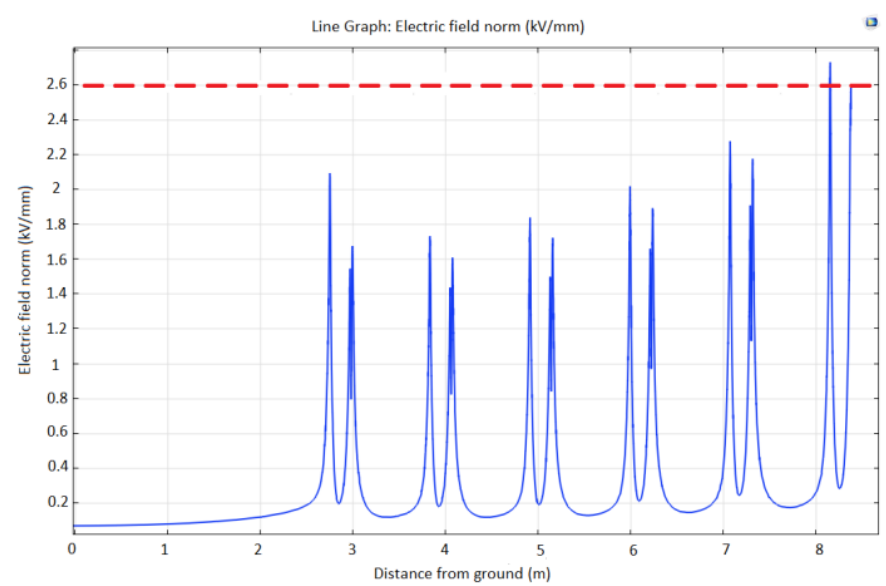

Fig. 7. Electric field variation along $500 \mathrm{kV}$ AC capacitor bank without corona rings $(\mathrm{kV} / \mathrm{mm}-\mathrm{m})$. Dashed red line presents the corona inception threshold $(2.6 \mathrm{kV} / \mathrm{mm})$

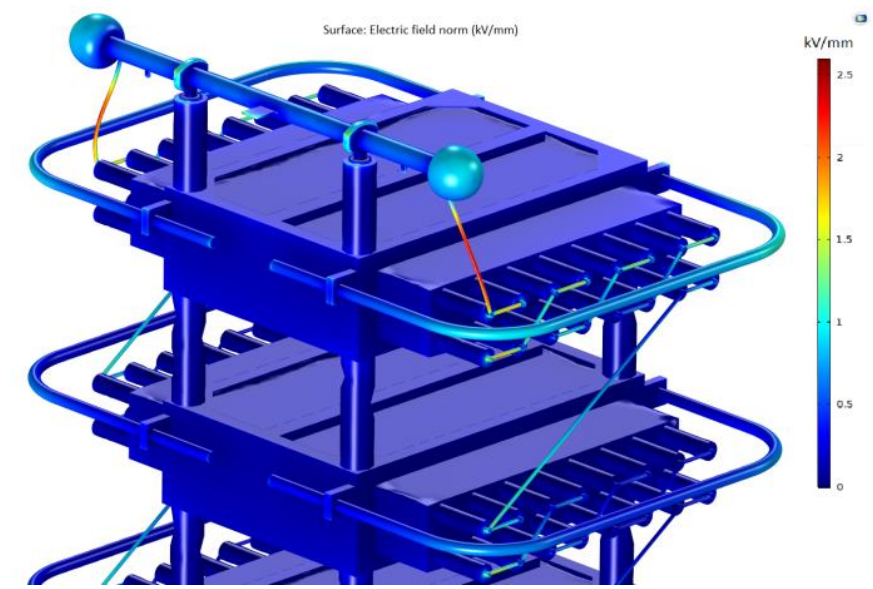

Fig. 8. Electric field distribution for $500 \mathrm{kV}$ AC capacitor bank with corona rings

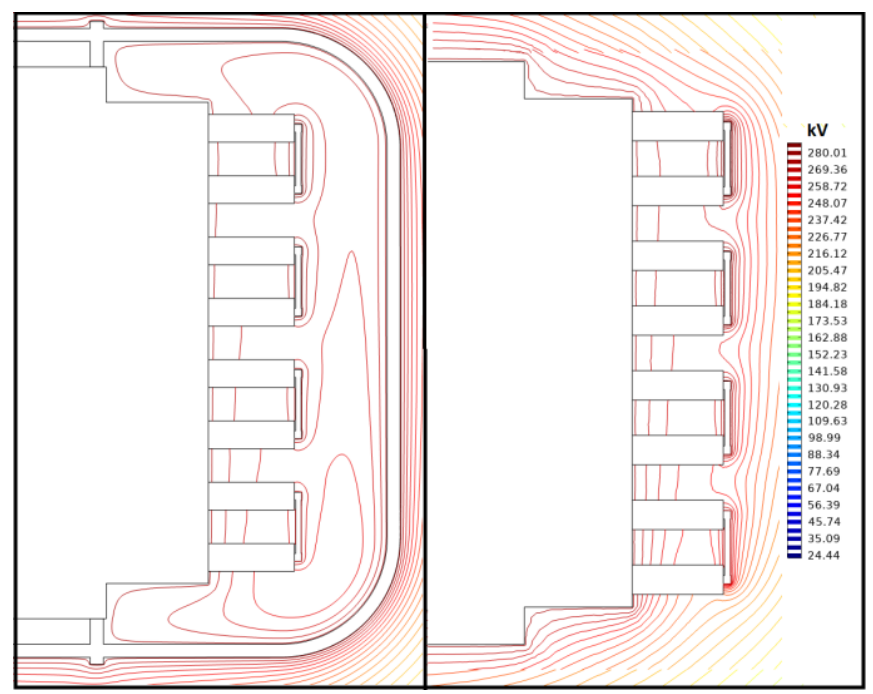

Fig. 9. Equipotential lines at the top level of $500 \mathrm{kV}$ AC bank with corona ring (left) and without the ring (right)

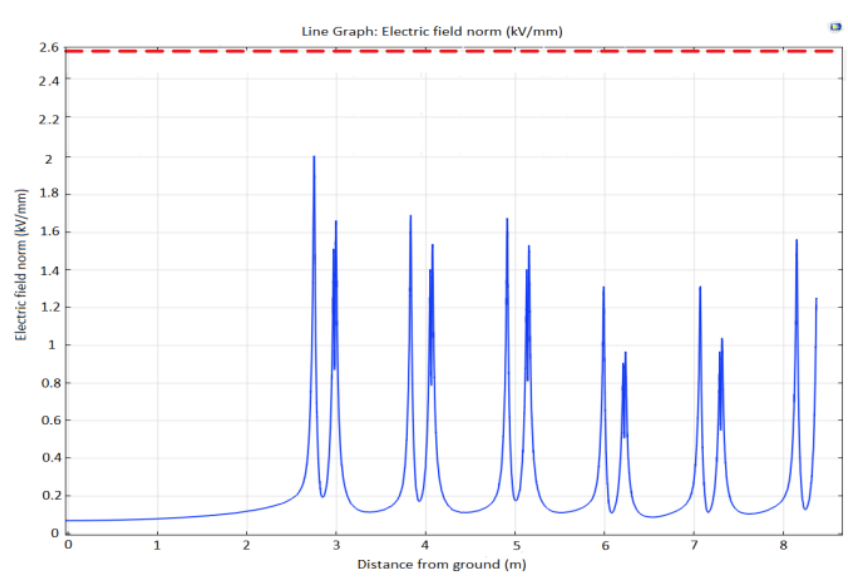

Fig. 10. Electric field variation along $500 \mathrm{kV}$ AC capacitor bank with corona rings $(\mathrm{kV} / \mathrm{mm}-\mathrm{m})$. Dashed red line presents the corona inception threshold (2.6 $\mathrm{kV} / \mathrm{mm})$ 
proposed ring as well as reduction of electric field (below $2.6 \mathrm{kV} / \mathrm{mm}$ ). Furthermore, maintenance maneuverability is enhanced since the proposed solution does not interfere with the maintenance operation. In other words, this solution creates an opportunity to reach the units without disassembling the rings. Moreover, electric field variation figure along $500 \mathrm{kV}$ AC bank shows considerable low E-field values as presented in Fig. 15 and Fig. 16.

2. For capacitor banks with high voltage levels that full ring does not have sufficient effect on electric field gradient reduction (specifically at the toplevel), HV busbar structure and location should be modified as shown in Fig. 18. Since spheres create even distribution of the E-field and reduce the intensities, they are placed near the top cables by bending HV busbar gradually towards the terminals. It is noteworthy to mention that, proper air clearance distance is considered according to the IEC 60871-1 standard [28]. Furthermore, two HV busbars are required rather than one, in order to cover all the areas. Fig. 18, demonstrates employed solutions for $500 \mathrm{kV}$ DC bank which is the combination of proposed ring as well as bent HV busbars. Electric field values are considerably below the limit as shown in Fig. 19.

\section{CONCLUSION}

Corona discharge behavior on AC and DC capacitor banks were investigated and verification models are created. All the models simulated with three scenarios, with suppression rings, without suppression rings, and with proposed solutions. Critical areas with high electric field values are identified and optimal solutions introduced based on previous results. Significant electric field differences were identified between the models with, and without rings which verifies the effect of suppression rings. However, existence of a complete corona ring at top-level does not guarantee the electric field reduction and corona discharge elimination in high voltage banks such as, $500 \mathrm{kV}$ DC case. Besides, full rings can interfere with maintenance operation due to the necessity of complete disassembly which can affect time and costs. Low flexibility is another major drawback of these rings i.e., using a full ring to cover the entire area, while only a part of the region has high field values, makes it less flexible. Therefore, rings that cover side terminals and two bent HV busbars at the top-level are proposed as an optimal solution. Results have shown the efficiency of the proposals as well as maintenance maneuverability enhancement due to the created free spaces in front of the units.

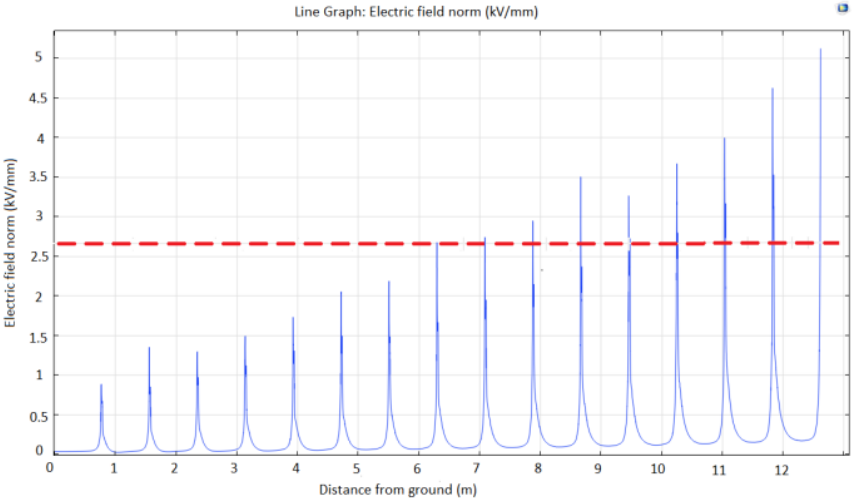

Fig. 11. Electric field variation along $500 \mathrm{kV}$ DC capacitor bank without corona rings $(\mathrm{kV} / \mathrm{mm}-\mathrm{m})$. Dashed red line presents the corona inception threshold $(2.6 \mathrm{kV} / \mathrm{mm})$. Top nine levels show considerable high E-field values.

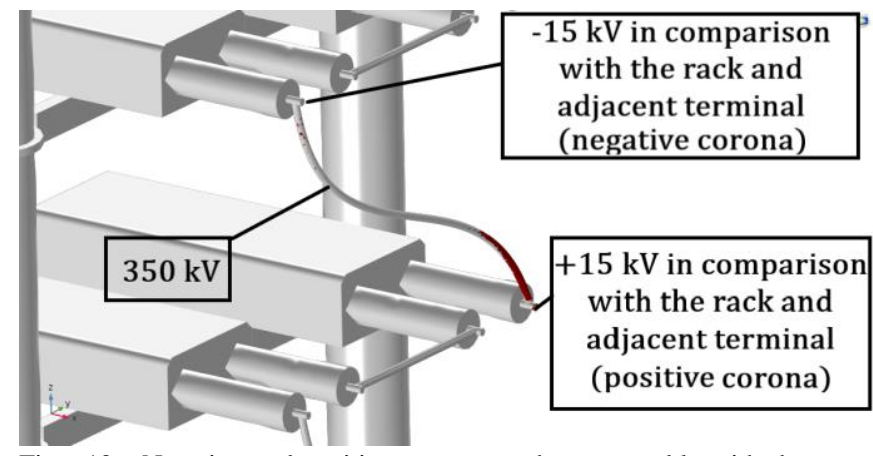

Fig. 12. Negative and positive corona on the same cable with the same potential between two different levels in the capacitor bank.

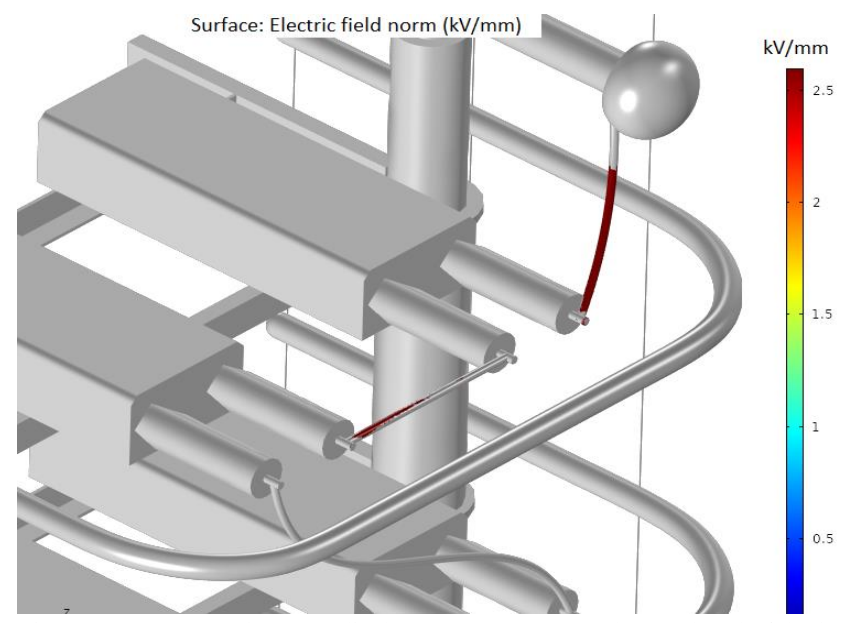

Fig. 13. Unexpected corona discharge appearance at the top-level of the bank with presence of the suppression ring 


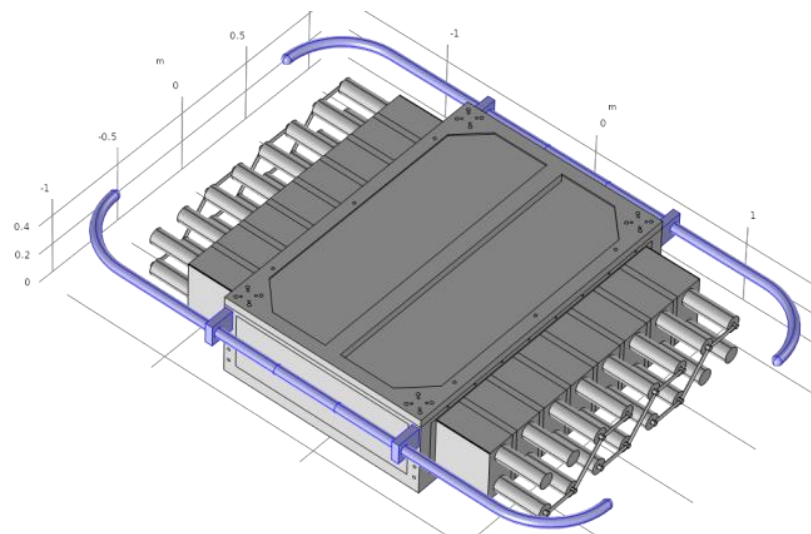

Fig. 14. Proposed solution for ring optimization, highlighted with blue color.

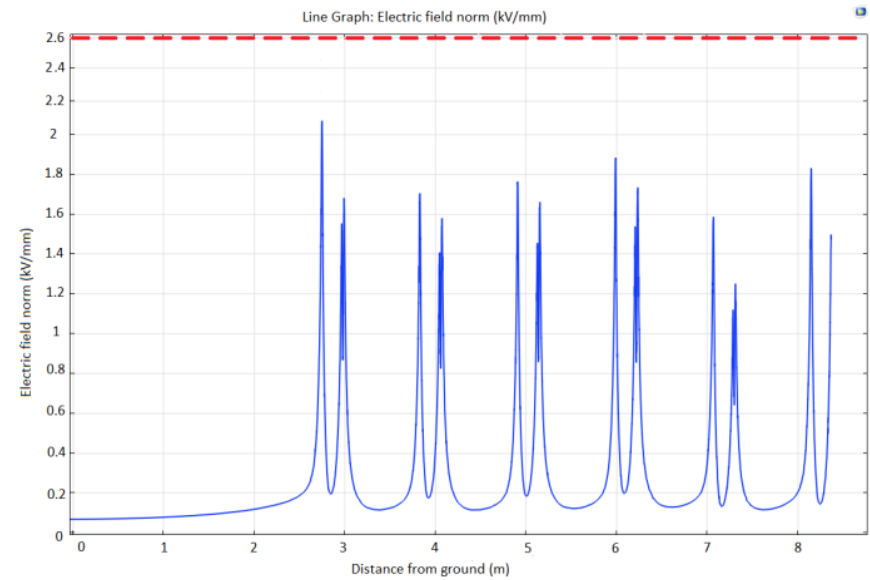

Fig. 15. Electric field variation for the proposed solution in $500 \mathrm{kV}$ AC bank. Dashed red line presents the corona inception threshold $(2.6 \mathrm{kV} / \mathrm{mm})$.

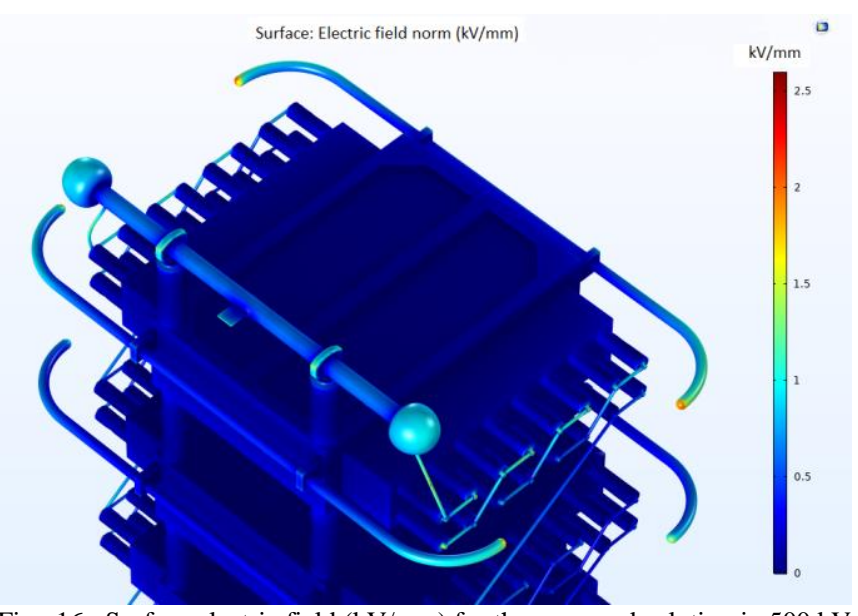

Fig. 16. Surface electric field $(\mathrm{kV} / \mathrm{mm})$ for the proposed solution in $500 \mathrm{kV}$ AC bank.

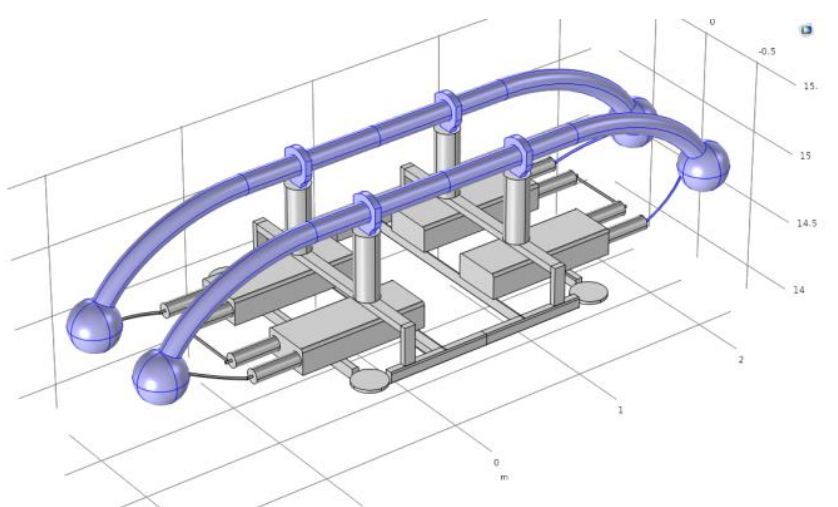

Fig. 17. Proposed solution for top-level where intensive electric field appears in high voltages, highlighted with blue color.

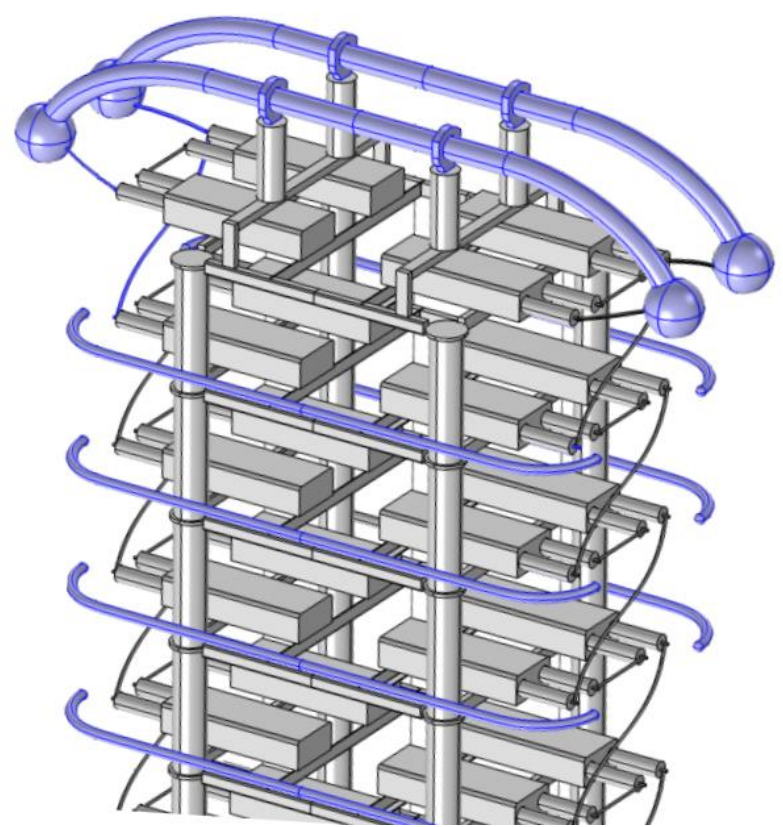

Fig. 18. Proposed solutions for $500 \mathrm{kV}$ DC bank, highlighted with blue color.

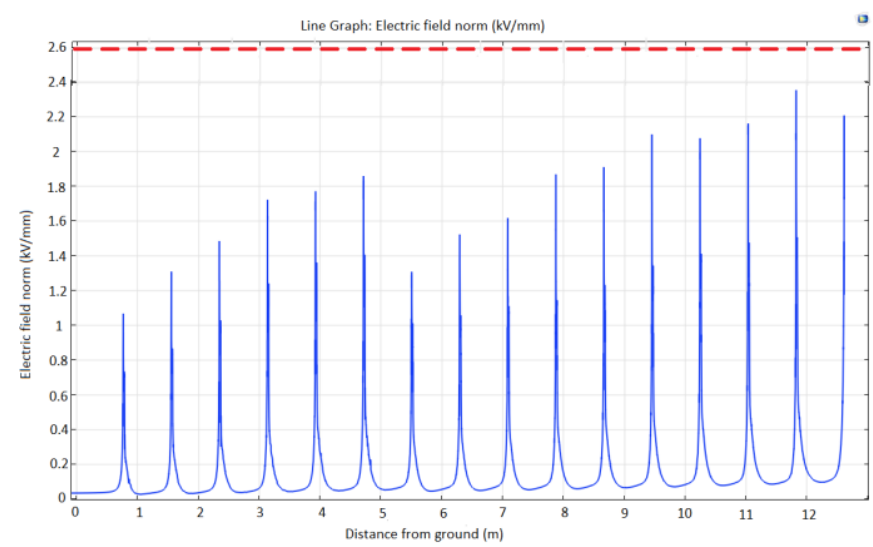

Fig. 19. Electric field variation for the proposed solutions in $500 \mathrm{kV}$ DC bank. Dashed red line presents the corona inception threshold $(2.6 \mathrm{kV} / \mathrm{mm})$ 


\section{REFERENCES}

[1]"IEEE Std 1036-1992, IEEE Guide for Application of Shunt Power Capacitors," p. 51.

[2]Lipeng Liu, "Physics of Electrical Discharge Transitions in Air," 2017, doi: 10.13140/RG.2.2.36391.96162.

[3]E. Kuffel, W. S. Zaengl, and J. Kuffel, High voltage engineering: fundamentals, 2nd ed. Oxford; Boston: Butterworth-Heinemann, 2000.

[4]W. Sima, F. P. Espino-Cortes, E. A. Cherney, and S. H. Jayaram, "Optimization of corona ring design for long-rod insulators using FEM based computational analysis," in Conference Record of the 2004 IEEE International Symposium on Electrical Insulation, Sep. 2004, pp. 480-483, doi: 10.1109/ELINSL.2004.1380655.

[5]S. Ilhan and A. Ozdemir, " $380 \mathrm{kV}$ corona ring optimization for $\mathrm{AC}$ voltages," IEEE Trans. Dielectr. Electr. Insul., vol. 18, no. 2, pp. 408-417, Apr. 2011, doi: 10.1109/TDEI.2011.5739444.

[6]J. Li, Z. Peng, Y. Feng, X. Fu, and T. Xie, "Electric field calculation and grading ring optimization of composite insulator for $500 \mathrm{kV}$ AC transmission lines," in 2010 10th IEEE International Conference on Solid Dielectrics, Jul. 2010, pp. 1-4, doi: 10.1109/ICSD.2010.5567918.

[7]T. Doshi, R. S. Gorur, and J. Hunt, "Electric field computation of composite line insulators up to $1200 \mathrm{kV}$ AC," IEEE Trans. Dielectr. Electr. Insul., vol. 18, no. 3, pp. 861-867, Jun. 2011, doi: 10.1109/TDEI.2011.5931075.

[8]N. Mohan, "Using Gaussian Process Model," p. 141.

[9]H. Terrab and A. Kara, "Parameters design optimization of $230 \mathrm{kV}$ corona ring based on electric field analysis and response surface methodology," Electr. Power Syst. Res., vol. 163, pp. 782-788, Oct. 2018, doi: 10.1016/j.epsr.2017.06.002.

[10] R. M. R. Barros, E. G. da Costa, T. V. Ferreira, J. F. Araujo, and F. L. M. Andrade, "A new approach for optimal design of corona ring," in 2016 IEEE International Power Modulator and High Voltage Conference (IPMHVC), San Francisco, CA, USA, Jul. 2016, pp. 684-687, doi: 10.1109/IPMHVC.2016.8012894.

[11] C.-C. Hsu and C.-Y. Wu, "Electrical characterization of the glow-to-arc transition of an atmospheric pressure pulsed arc jet," J. Phys. Appl. Phys., vol. 42, no. 21 , p. 215202, Nov. 2009, doi: 10.1088/0022-3727/42/21/215202.

[12] "Electrical Breakdown in Gases," in Foundations of Pulsed Power Technology, Hoboken, NJ, USA: John Wiley \& Sons, Inc., 2017, pp. 369-438. [13] S. Singh, Y. Serdyuk, and R. Summer, "Streamer propagation in air in non-axially symmetric electric field," p. 6.

[14] M. Khalifa and M. Abdel-Salam, "TIPROVED CALCULATION OF CORONA PHJI," p. 7.

[15] E. Fuchs and M. A. S. Masoum, Power Quality in Power Systems and Electrical Machines. San Diego, UNITED STATES: Elsevier Science \& Technology, 2008.

[16] X. Bian, L. Wang, J. K. Macalpine, Z. Guan, J. Hui, and Y. Chen, "Positive corona inception voltages and corona currents for air at various pressures and humidities," IEEE Trans. Dielectr. Electr. Insul., vol. 17, no. 1, pp. 63-70, Feb. 2010, doi: 10.1109/TDEI.2010.5412003.

[17] J.-S. Chang, P. A. Lawless, and T. Yamamoto, "Corona discharge processes," IEEE Trans. Plasma Sci., vol. 19, no. 6, pp. 1152-1166, Dec. 1991 , doi: 10.1109/27.125038.

[18] L. Arevalo and V. Cooray, "Preliminary study on the modelling of negative leader discharges," J. Phys. Appl. Phys., vol. 44, no. 31, p. 315204, Aug. 2011, doi: 10.1088/0022-3727/44/31/315204.

[19] J.-R. Riba, A. Morosini, and F. Capelli, "Comparative Study of AC and Positive and Negative DC Visual Corona for Sphere-Plane Gaps in Atmospheric Air," Energies, vol. 11, no. 10, p. 2671, Oct. 2018, doi: 10.3390/en11102671.

[20] G. Eriksson, "Easy Evaluation of Streamer Discharge Criteria," p. 7.

[21] D. F. Ariza, O. R. Montero, F. J. Roman, and O. F. Escobar, "Influence of the wind for positive corona in a single-needle-electrode on thunderstormlike conditions," in 2011 International Symposium on Lightning Protection, Fortaleza, Brazil, Oct. 2011, pp. 101-106, doi: 10.1109/SIPDA.2011.6088455. [22] J. K. Chan, J. Kuffel, G. C. Sibilant, and J. Bell, "METHODOLOGY FOR HVDC CORONA TESTS,” p. 12.

[23] L. B. Loeb and J. M. Meek, "The Mechanism of Spark Discharge in Air at Atmospheric Pressure. I," J. Appl. Phys., vol. 11, no. 6, pp. 438-447, Jun. 1940, doi: 10.1063/1.1712792.

[24] K. T. A. L. Burm, "Calculation of the Townsend Discharge Coefficients and the Paschen Curve Coefficients," Contrib. Plasma Phys., vol. 47, no. 3, pp. 177-182, May 2007, doi: 10.1002/ctpp.200710025.

[25] X. Zhang et al., "Experimental investigation on humidity effects on the variations of positive DC corona discharge," in 2016 IEEE International Power
Modulator and High Voltage Conference (IPMHVC), San Francisco, CA, USA, Jul. 2016, pp. 598-602, doi: 10.1109/IPMHVC.2016.8012772.

[26] H. Ryzko, "Drift velocity of electrons and ions in dry and humid air and in water vapour," Proc. Phys. Soc., vol. 85, no. 6, pp. 1283-1295, Jun. 1965, doi: 10.1088/0370-1328/85/6/327.

[27] "Spark Discharge by Eduard M. Bazelyan, Yuri P. Raizer: New Hardcover (1997) 1. | Ergodebooks." https://www.abebooks.co.uk/SparkDischarge-Eduard-Bazelyan-Yuri-Raizer/11800566989/bd (accessed Dec. 18, 2020).

[28] International Electrotechnical Commission, International Electrotechnical Commission, and Technical Committee 33, Shunt capacitors for a.c. power systems having a rated voltage above $1000 \mathrm{~V}$. Part 1, Part 1, 2014. 